\title{
Kalman Filtering based Channel Estimation for MIMO-OFDM
}

\author{
Ashish Kr. Gupta \\ Dehradun Institute of \\ Technology, \\ Dehradun
}

\author{
Mukesh Pathela \\ Dehradun Institute of \\ Technology, \\ Dehradun
}

\author{
Arun Kumar \\ Doon College of Engineering \\ \&Technology, \\ Dehradun
}

\begin{abstract}
In this paper, Kalman filtering is used for MIMO-OFDM channel estimation. First we uses the MIMO-OFDM Training sequences, the current channel response at the receiving end, and then use the Kalman filter method to estimate the channel response. The measure and tracking, using the Jakes channel transmission model. Through simulation can be seen, and compared to other channel estimation method proposed in this paper. Method can fast-track the time-varying channel where the channel response changes very quickly. At the same time, without excessive insertion of training sequences and pilot symbols, the performance of more greatly improved.
\end{abstract}

\section{Keywords}

MIMO-OFDM, Kalman Filtering, Jakes Model, AR process, Channel Estimation

\section{INTRODUCTION}

With the increasingly high demand for wireless communication systems, the rate and frequency Spectrum resources are increasingly strained and the requirements of the wireless communication system spectral efficiency increasingly high. Here we use OFDM because of its high resistance to multipath fading characteristics as well as more High spectral efficiency of a large number of applications. Meanwhile, with the multi-antenna Application of the system spectral efficiency has been greatly improved. MIMO and OFDM technology together can significantly improve the spectrum utilization and transmission rate, many scholars and research in the next-generation wireless communication systems will be widely used.

Wireless communication systems due to the special environment of transmission modulation signal, the transfer process will be a lot of interference. Accurately estimate the channel at the receiving end, response parameters are particularly important. In the slow fading channel, relatively slow changes in the channel, the channel estimation by training sequence can be used. In the fast fading channel, the channel fading changes rapidly, required after the sender sends a training sequence at the receiving end tracking and forecasting of channel Parameter changes. Traditional channel estimation methods in two ways: blind estimation methods and guidelines frequency of the auxiliary estimation method. Blind estimation methods use the internal nodes of the data structure, data signals require a longer and more complex calculation for Speed time-varying wireless channel does not apply. [1], gives a multi households OFDM system related to blind channel estimation method; literature [2] gives the basic principles of the blind channel estimation; [3] gives a utilization virtual carrier blind channel estimation methods, and other major research are: $[4,5,6]$. Pilot Symbol Aided channel estimation method is mainly used in a few. According to insert a certain number of time domain or frequency domain known data stream (conductivity Frequency), each pilot subchannel should be ring the channel periodically to detect at the receiving end, and then be interpolated to the channel estimation, so that you can known point on the sample value of the channel response to estimate the integrity of the entire channel response. can be divided into onedimensional and two-dimension according to the different pilot distribution.

The key findings are: [7, 8, and 9]. For the slow decay channel blind estimation methods and pilot-assisted estimation methods can achieve better performance. But for fast time-varying channels, blind estimation method channel changes in the situation due to its computational complexity is enormous, and therefore can not be used in rapid response conditions. Pilot assisted estimation methods need to insert too much pilot sequence, leading caused the system transmission rate is too low. [10] is given based on improved Kalman filter channel estimation method, the AR model Rayleigh channel modeling, tracking and estimation of AR parameters channel tracking and response.

This paper presents a Kalman filter channel tracking algorithm, frequency signal with the sending end and guide channel at the receiving end response, conducted with the use of Kalman filtering method for fast time-varying channel, tracking and estimation. The advantages of the method for fast time-varying channel, do not need to additional pilot signal, only need to use the original pilot sequence, algorithm complexity and accuracy have a large performance increase. This article first discuss the MIMO-OFDM system model and MIMO-OFDM Pilot sequence inserted in the system rules were discussed, and optimal pilot sequence inserted principles, and then use of the Kalman filter for channel estimates are discussed and the method is given to other parties, and finally through the simulation.

2. MIMO-OFDM system model [11]: MIMOOFDM system block diagram shown in Figure 1. We assume that at the sending end the no. of antennas is $T_{N}$ and at the receiving end the no. of receiving antennas is $R_{N}$. Data flowing through the coding and divided into $T_{N}$ different symbol block sent from different antennas. Each Send signals used OFDM modulation. The cyclic prefix length $\mathrm{N}_{\mathrm{g}}$ larger than the channel multipath delay $\mathrm{L}$. At the receiving end each antenna receives a superposition of transmitted signal from $T_{N}$ transmitting antennas. 


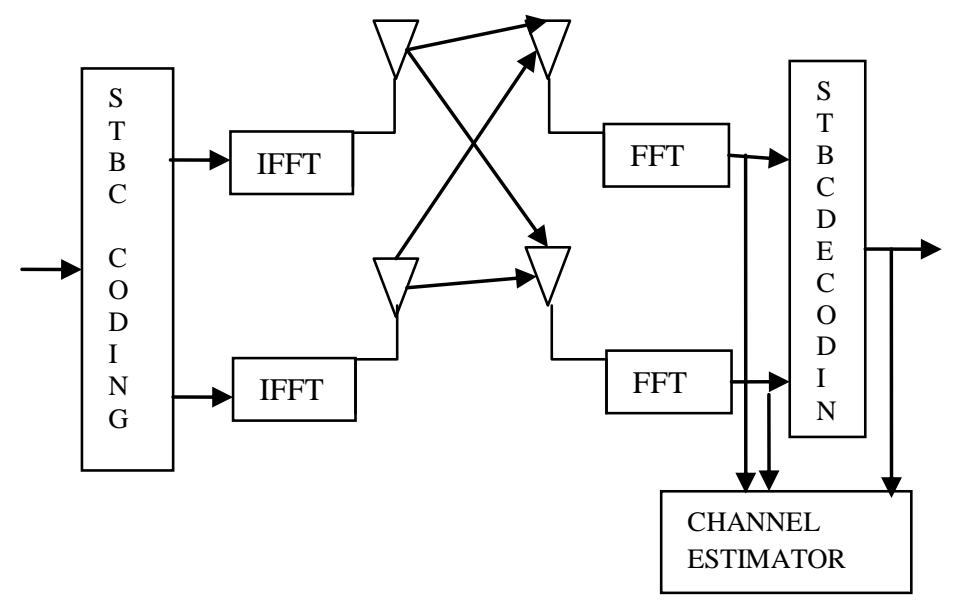

Figure 1: A 2×2 MIMO System model

The received signal at the receiver by the $\mathrm{n}_{\mathrm{th}}$ receiving antenna can be expressed as:

$Y_{n, k}=\sum_{m=1}^{N_{r}} H_{n, m, k} X_{m, k}+V_{n, k}$

Where $H_{n, m, k}$ is the channel frequency response between $\mathrm{m}_{\mathrm{th}}$ transmitting antenna to the $\mathrm{n}_{\mathrm{th}}$ receiving antenna in the $\mathrm{k}_{\mathrm{th}}$ subchannel, $X_{\mathrm{m}, \mathrm{k}}$ represents the frequency domain signal from the $\mathrm{m}_{\mathrm{th}}$ transmitting antenna, $\mathrm{V}_{\mathrm{n}, \mathrm{k}}$ is the additive white Gaussian noise from $\mathrm{n}_{\mathrm{th}}$ receiving antenna with mean 0 and variance $(\delta) 2$. Vector representation of the signal from $\mathrm{k}_{\mathrm{th}}$ subchannel of $n_{\mathrm{th}}$ receiving antenna is:

$$
\begin{aligned}
& Y_{k}=\left[Y_{1, k} Y_{2, k} \ldots \ldots Y_{N, k}\right]^{T}, \\
& Y_{k}=H_{k} X_{k}+V_{k}
\end{aligned}
$$

Where $H_{k}$ is the channel frequency response, $\mathrm{N} \times \mathrm{N}$ matrix of the $\mathrm{K}_{\mathrm{th}}$ subchannel of the MIMO and $X_{k}=\left[X_{1, k} X_{2, k} \ldots \ldots X_{M, k}\right]^{T}$ is the $\mathrm{k}_{\mathrm{th}}$ sub-channel $\mathrm{M}$ antenna excitation signal matrix.

Channel estimation to restore the data stream received signal decoding is done by using $\mathrm{N} \times \mathrm{N}$ receiver.

\section{Optimal Pilot Design: The Channel Estimation} first is calculated according to the received signal and the excitation signal pilot subchannel estimates $H_{k}$, the minimum variance estimation criterion:

$$
\widehat{H}_{k}=\widehat{Y}_{k} \widehat{X}_{k}
$$

Where $\hat{X}_{k}=X_{k}^{H}\left(X_{k} X_{k}^{H}\right)^{-1}$, substituting (2) into (3) to get $\widehat{H}_{k}=H_{k}+V_{k} \hat{X}_{k}$, the pilot subchannel estimates contrast as:

$$
\begin{aligned}
& E\left\|\widehat{H}_{k}-H_{k}\right\|^{2}=E\left\|V_{k} \hat{X}_{k}\right\|^{2}=\operatorname{tr}\left\{E\left[\left(\left(V_{k} \hat{X}_{k}\right)^{H}\left(V_{k} \hat{X}_{k}\right)\right)\right]\right\}= \\
& N \delta^{2} \operatorname{tr}\left\{\left(X_{k} X_{k}^{H}\right)^{-1}\right\}
\end{aligned}
$$

Where $E\left[V_{k}^{H} V_{k}\right]=N \delta^{2}$ is the square of the trace. Set up $\left\|X_{k}\right\|^{2}=\delta$ all the antennas in a pilot subchannels to send a letter No. of total power in the MIMO system regardless of the number of antennas, the total power protection $\left\|X_{k}\right\|^{2}=\left\{X_{k} X_{k}^{H}\right\}$, remain unchanged., set $\left(X_{k} X_{k}^{H}\right)^{-1}$ as special Intrinsic value $\lambda_{m}\left(m=1,2, \ldots \ldots N_{t}\right)$ then eigenvalue for $X_{k} X_{k}^{H}$ on $1 / \lambda_{m}\left(m=1,2, \ldots \ldots N_{t}\right)$ because of its Hermitian matrix, Trace equal to matrix eigenvalue, and thus channel estimation minimum Mean square error of the problem becomes:

$$
\sum_{m=1}^{N_{t}} 1 / \lambda_{m}=\rho
$$

Because the matrix is non-negative, so the eigenvalue is nonnegative. So the minimum can only occur when all eigenvalues are equal, $\left(\lambda_{m}=\lambda=M / \rho\right)$, the minimum mean square error:

$M M S E=\frac{1}{M N\left(E\left\|\widehat{H}_{k}-H_{k}\right\|^{2}\right)}=\sigma^{2} M / \rho$

(5) Style can be seen to reduce the number of transmit antennas to increase the transmit power Rate helps to reduce the mean square error. However, the transmitting antenna less the mass of the system transmission capacity will be reduced. Hermitian matrix $\boldsymbol{H}_{\boldsymbol{k}} \boldsymbol{X}_{\boldsymbol{k}}^{\boldsymbol{H}}$, feature decomposition $X_{k} X_{k}^{H}=$ $\Phi U \Omega \Phi^{\mathrm{H}}$, where $\mathrm{U}$ is constituted by the eigenvalue diagonal matrix, These feature values are the same, so $\boldsymbol{U}=\boldsymbol{\lambda} \boldsymbol{I}_{\boldsymbol{M}}$ is a unitary matrix, and $\boldsymbol{\Phi} \boldsymbol{\Phi}^{H}=\boldsymbol{\Phi}^{\mathrm{H}} \boldsymbol{\Phi}=\mathbf{I}$, so I can get: :

$$
\mathbf{X}_{\mathbf{k}} \mathbf{X}_{\mathbf{k}}^{\mathbf{H}}=\lambda \mathbf{I}_{\mathbf{M}}
$$

It can be seen from the above equation, the training sequence $X_{m, k}\left(m=1,2, \ldots \ldots \ldots \ldots . N_{t}\right) \quad$ must be Orthogonal to each other.

One of the simplest example is the training sequence, On the same subchannel only An antenna to send signals, the rest of the antenna to send the zero signal. With this Method, each antenna only one OFDM symbol block, training Antenna to send their respective training sequence

\section{Kalman Filtering Method: Introduction:}

Kalman filter state space to describe the system by the state party Process and measurement equations are composed. Its previous state estimates and the recent observational data to estimate the current value of the state variables and to the state variable. The amount of the estimate given in the form Kalman filter has the following two special Points: 1) algorithm is recursive, multi-dimensional stochastic process is estimated that Discrete Kalman algorithm is suitable for computer processing; 2) Kalman filtering do not need to know the value of all the past, the equation of state describes the state variables dynamic changes in the law. The error criterion used in this article Kalman filter is based on the minimum mean square error criterion.

5 Kalman filtering procedure: Assume that the channel is Rayleigh fading, Jakes channel model, the letter Channel correlation coefficient:

$E\left\{h_{n, l} h_{k, l}^{*}\right\}=r_{1}(n-k)=\sigma_{h_{l}}^{2} J_{0}\left(2 \pi f_{d} T_{s}(n-k)\right)$

Here $\sigma_{h_{l}}^{2}$ is channel-related power, $f_{d}$ is the Doppler shift.\& T for the sample Interval. $J_{0}$ is the first zero-order Bessel function. a stable with Machine process, we can use the finite AR model to be expressed. Therefore, the letter Tao response can be expressed as:

$h_{n, l}=\sum_{k=1}^{p} a_{k} h_{n-k, l}+v_{n}$

Where $\mathrm{p}$ and $\mathrm{k}$ are the order and coefficient of the AR process, $\boldsymbol{v}_{\boldsymbol{n}}$ is Zero mean and covariance Gaussian white noise $\sigma_{v}^{2}, \sigma_{v}^{2}$ is able through the Yule- Walker equations. In the first class of the AR model, $\sigma_{v}^{2}$ is given by the following formula:

$\sigma_{v}^{2}=\boldsymbol{r}_{\boldsymbol{l}}(\mathbf{0})\left(\mathbf{1}-\frac{r_{l}^{2}(\mathbf{1})}{r_{l}^{2}(\mathbf{0})}\right)=\boldsymbol{\sigma}_{\boldsymbol{h}_{l}}^{2}\left(\mathbf{1}-\boldsymbol{J}_{\mathbf{0}}^{2}\left(2 \pi f_{d} T_{s}\right)\right.$

Assume that the fading channel as an independent and identically distributed, and consider only the first An AR model, the channel response can be expressed as: 


$$
\begin{aligned}
& h_{n}=a h_{n-1}+v_{n} \\
& y_{n}=x_{n}^{T} h_{n}+w_{n},
\end{aligned}
$$

Now as defined in the equation to the measured value, the establishment of the measurement equation, and iteration, follow these steps:

1) to establish the measurement equation:

$$
y(n)=x_{n}^{T}(n)+v(n)
$$

2) to strike a measurement error:

$$
e_{n}=y_{n}-\hat{y}_{n}=y_{n}-x_{n}^{T} \hat{h}_{n}
$$

3) calculate the minimum predict MSE:

$$
r_{n}=\sigma_{n}^{2}+X_{n}^{T} P_{n} X_{n}^{*}
$$

4) Calculate the Kalman gain:

$$
k_{n}=a P_{n} X_{n}^{*} r_{n}^{-1}
$$

5) The amendment to the measured value:

$$
\hat{h}_{n+1}=a \hat{h}_{n}+k_{n} e_{n}
$$

6) to calculate the minimum the MSE:

$$
P_{n+1}=a\left(a I-k_{n} X_{n}^{T}\right) P_{n}+Q_{n+1}
$$

Where $Q_{n}$ is the system state noise covariance matrix Initialize h to $0 . P_{n}$ for h steady-state covariance calculated, said h Kalman estimates.

Repeat the above steps can obtained the estimated value response by the channel. The obtained Pilot channel estimation signal, can be obtained by quadratic interpolation processing $r$ transmit antennas to multiple sub-carrier of the channel between the $\mathrm{q}$ receive antennas frequency response of the estimated value.

\section{The simulation}

Select $2 \times 2$ multiple input multiple output antenna channel simulation using OFDM system parameters are as follows. Assume that the overall channel average power transmission Gain in the time of transfer a burst channel remains unchanged. Channel estimation mean square error is the average of all input and output channel. Information transfer rate is $20 \mathrm{MHz}$; the carrier frequency is $2 \mathrm{GHz}$ of mobile communications for background, the choice of the ITU-R M.1225 channel A model [ITU-R, \# 252]. In Rayleigh distribution Channel the magnitude of each path, the phase is subject to uniform points Cloth, and the channel energy normalized (Table 1)

Table 1: Simulation environment

\begin{tabular}{|l|l|}
\hline Parameter & value \\
\hline $\begin{array}{l}\text { The number of data } \\
\text { subchannels }\end{array}$ & 48 \\
\hline Number of subchannels pilot & 4 \\
\hline The total subchannel & 64 \\
\hline $\begin{array}{l}\text { The subchannel frequency } \\
\text { response }\end{array}$ & $0.3125 \mathrm{MHz}(20 \mathrm{MHz} / 64)$ \\
\hline The IFFT / the FFT cycle & $3.2 \mu \mathrm{s}$ \\
\hline Guard interval length & $0.8 \mu \mathrm{s}$ \\
\hline OFDM symbol block length & 4.0 \\
\hline $\begin{array}{l}\text { Short training sequence } \\
\text { length }\end{array}$ & 8 \\
\hline $\begin{array}{l}\text { Long training sequence } \\
\text { length }\end{array}$ & 8 \\
\hline
\end{tabular}

\begin{tabular}{|l|l|}
\hline Relative time delay $(\mathrm{ns})$ & Average power $(\mathrm{dB})$ \\
\hline 0 & 0.0 \\
\hline 310 & -1.0 \\
\hline 710 & -9.0 \\
\hline 1090 & -10.0 \\
\hline 1730 & -15.0 \\
\hline 2510 & -20.0 \\
\hline
\end{tabular}

Figure 2 shows a comparison between the kalman filter and RLS. From Figure we can see that compared to RLS, Kalman filter-based method The method has great performance.

Figure 3 shows the maximum Doppler frequency shift fdTs $=$ 0.001 when Kalman Estimated and the performance comparison between the linear iterative estimation and DFT iterative estimation.

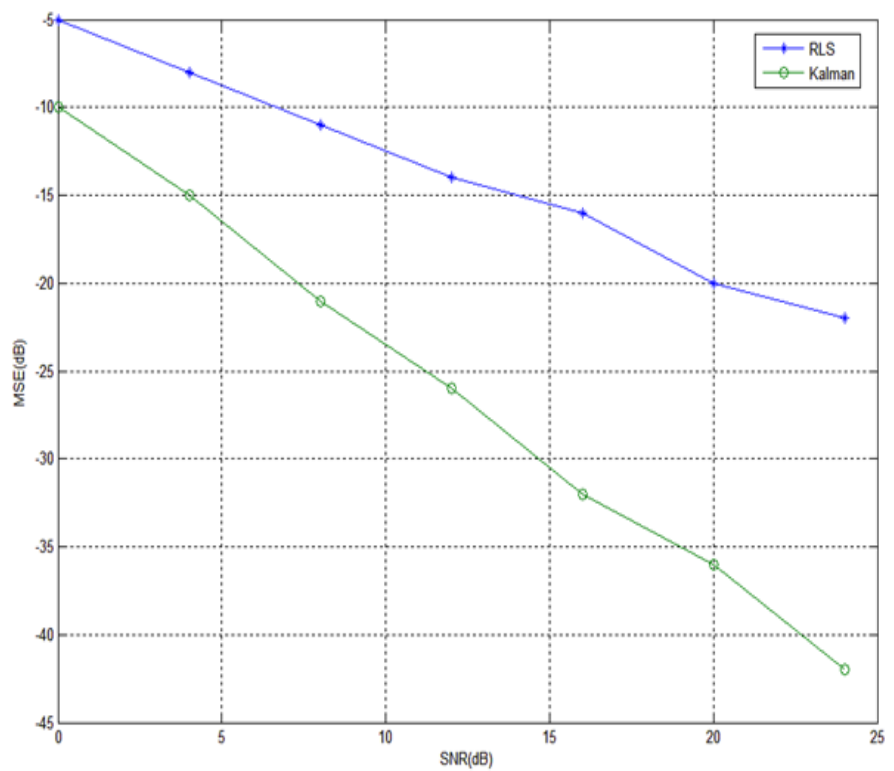

Figure 2: Comparison of Kalman and RLS

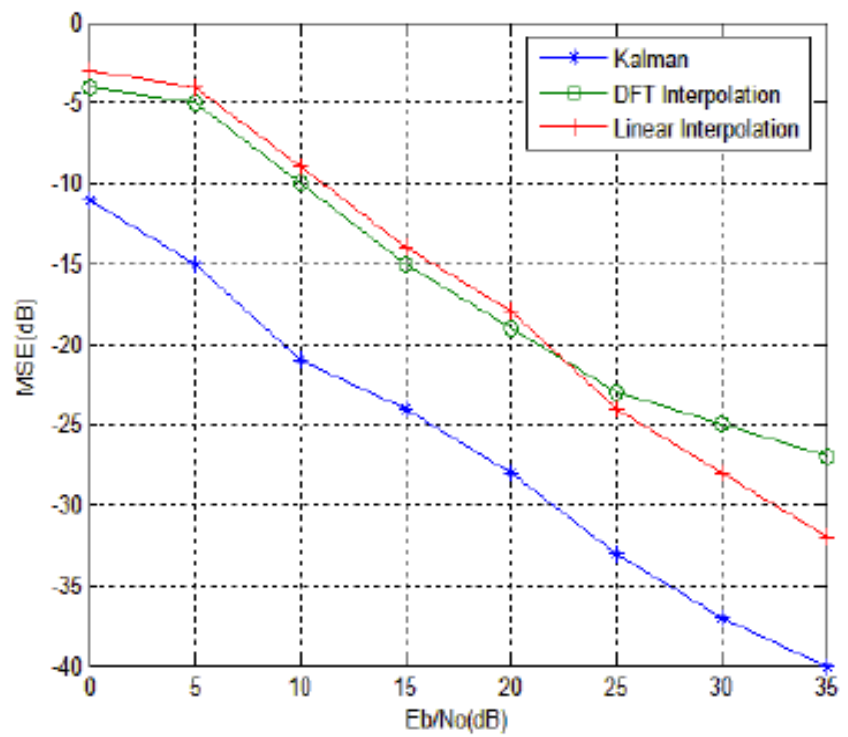

Figure 3: Comparison of Kalman and LI and DFT when fdTs $=0.001$.

Figure 4 shows the maximum Doppler frequency shift when $\mathrm{fdTs}=0.01$, Comparison between the Kalman estimation and linear iterative estimation and DFT estimated. 
It can be seen from the two diagrams of this paper are able to better track changes in the channel.

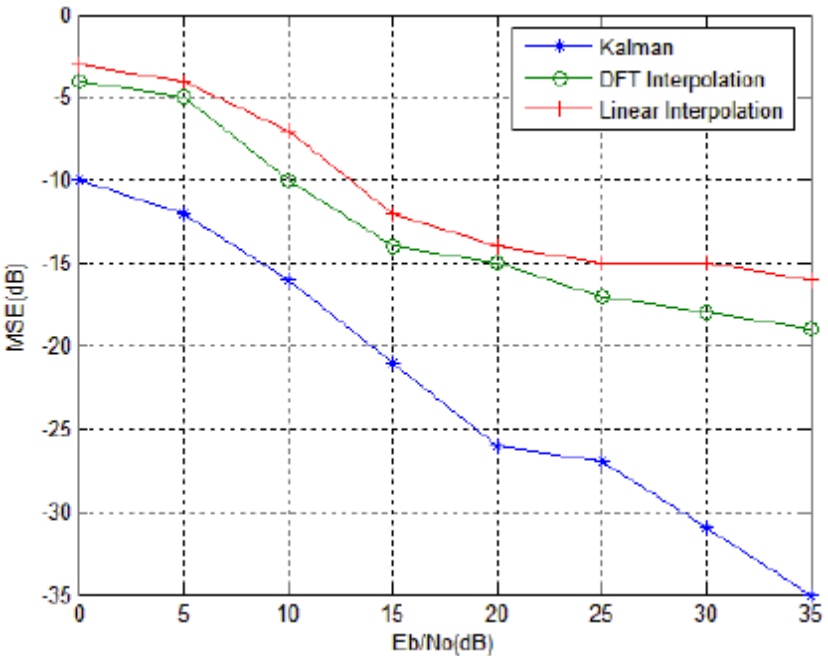

Figure 4: Comparison of Kalman and LI and DFT when fdTs $=0.01$

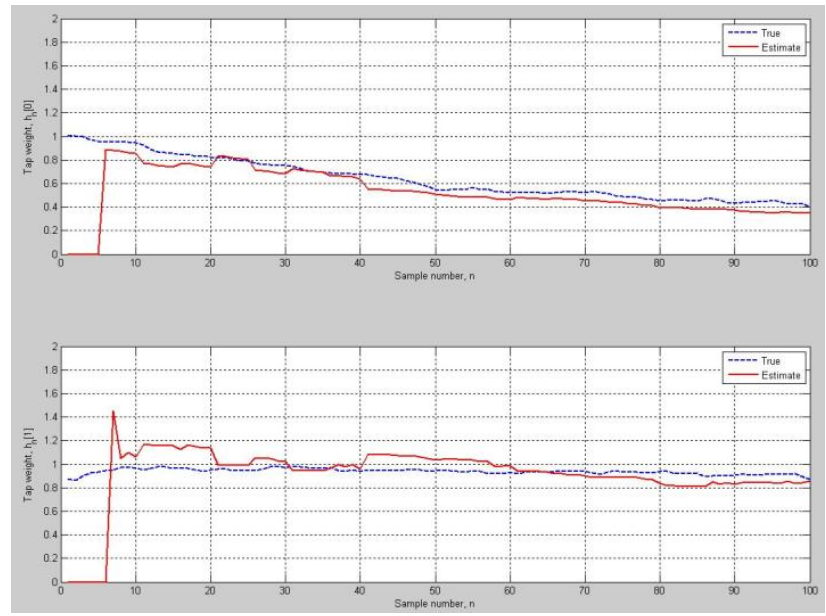

Figure 5: Realization of TDL coefficients

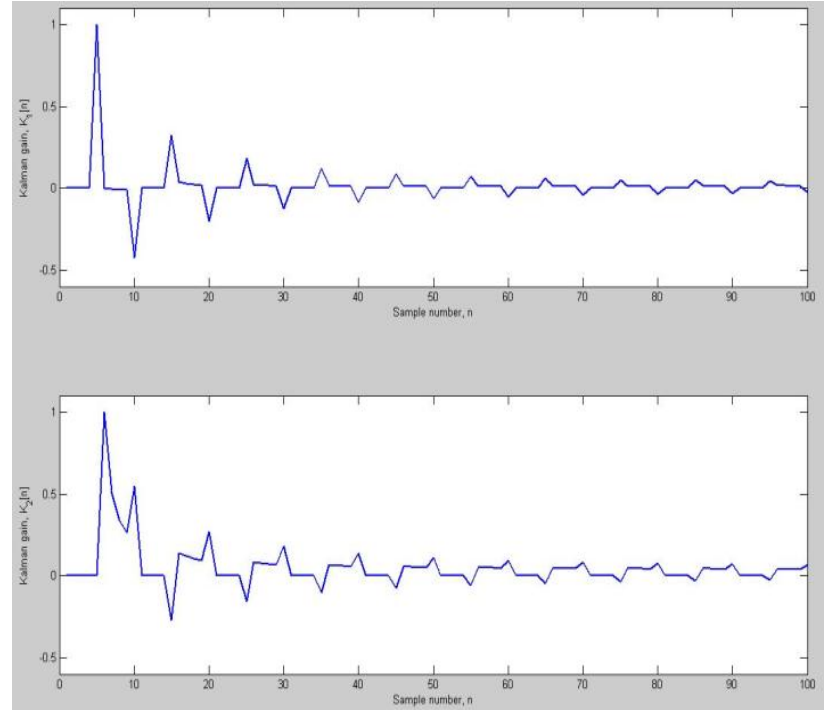

Figure 6: Kalman Gain obtained against Samples

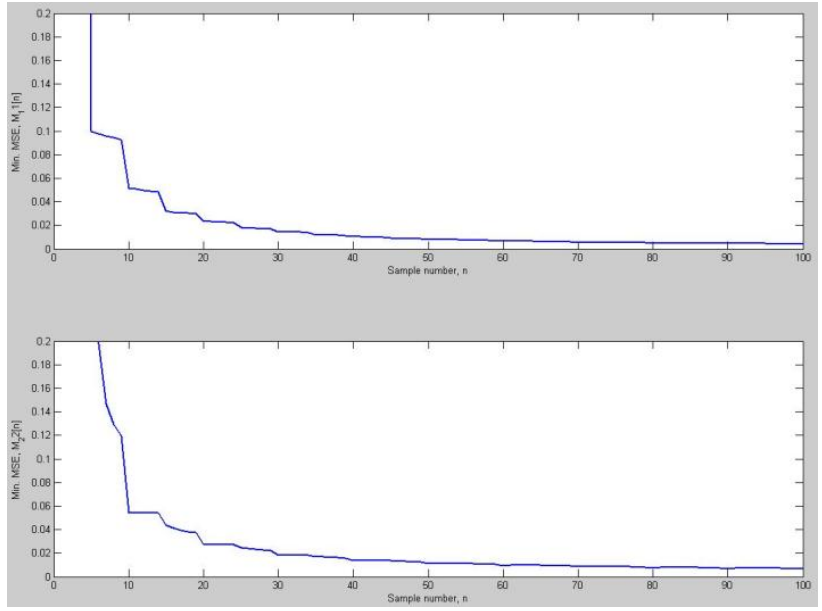

Figure 7: MMSE against the training sequence.

\section{Conclusion}

Pilot carrier channel estimation methods offer low complexity and good performance and are thus quite widely used in communications systems today. But they are also wasteful of bandwidth since they use long training sequences to estimate the channel. This paper presents a method of improving the channel estimate without increasing the length of the training sequence. This method uses the underlying channel model and the available data based estimate, to implement the channel estimation algorithm in the form of a Kalman filter. In this method based on Kalman filtering MIMO-OFDM Channel estimation method using the AR process is built on the Jakes model channel mode, using the training sequence in the system can track a time-varying channel changes. The Kalman filter based channel estimator leads to a significant gain in performance as compared to the data-only estimator. The Kalman filter also allows us to predict the state of the before the frame is actually received

As shown in figure 5,6, and 7 Simulation data, the method compared to the general channel estimation method in terms of performance there is a greater increase and the ability to fast track the channel changes. In Doppler shift to larger, a good estimate of the channel.

\section{References}

[1] S. Yatawatta and A. P. Petropulu, Blind channel estimation in mimo-ofdm systems with multiuser interference, Signal Processing, IEEE Transactions on [see also Acoustics, Speech, and Signal Processing, IEEE Transactions on] 54 (2006), no. 3, 1054-1068.

[2] C. Shin, W. Robert Jr and E. J. Powers, Blind channel estimation for mimo-ofdm systems, Vehicular Technology, IEEE Transactions on 56 (2007), no. 2, 670685.

[3] C. Shin and E. J. Powers, Blind channel estimation for mimo-ofdm systems using virtual carriers, 2004, p.^pp. 2465-2469 Vol.2464.

[4] C. Gao, Z. Ming, Z. Shidong and Y. Yan, Blind channel estimation algorithm for mimo-ofdm systems, Electronics letters 39 (2003), no. 19, 1420-1422.

[5] B. Wei, H. Chen, J. Ling-ge and Z. Hong-wen, Blind channel estimation in mimo-ofdm systems, 2002, . $^{\wedge} \mathrm{pp}$. 317-321 vol.311. 
[6] C. Xi, A. R. Leyman and F. Jun, Blind channel estimation for linearly precoded mimo-ofdm, 2006, p.^pp. IV-381-IV-384.

[7] H. A. C. Habib Senol, Erdal Panayirci, Pilot-aided bayesian mmse channel estimation for ofdm systems: Algorithm and performance analysis, (2004).

[8] W. Lidong and L. Dongmin, A pilot embedded scheme for doublyselective mimo-ofdm channel estimation, 2006, p.^pp. $1-5$.

[9] J. Wang and K. Araki, Pilot-symbol aided channel estimation in spatially correlated multiuser mimo-ofdm channels, 2004, p.^^p. 33-37 Vol. 31.
[10] K. Y. Han, S. W. Lee, J. S. Lim and K. M. Sung, Channel estimation for ofdm with fast fading channels by modified kalman filter, Consumer Electronics, IEEE Transactions on 50 (2004), no. 2, 443-449.

[11] Branka Vucetic, Jinhong Yuan, Space-Time Coding, John Wiley 2003.

[12] Zhou Jian, Channel Estimation for MIMO-OFDM System, Journal of Nanjing University of Posts and Telecommunications (Natural Science),26(2006),No.5, 1116 .

[13] ITU-R, Recommendation itr-r m.1225 guidelines for evaluation of radio transmission technologies for imt2000.Radio transmission technologies for imt-2000. 